\title{
Hepatoid thymic carcinoma: a case report of a rare subtype of thymic carcinoma
}

\author{
Ji-Seon Jeong $^{1 *}$, Hyo Jeong Kang ${ }^{1 *}$, Uiree Jo ${ }^{1}$, Min Jeong Song ${ }^{2}$, Soon Yeol Nam ${ }^{3}$, Joon Seon Song ${ }^{1}$ \\ 'Department of Pathology, Asan Medical Center, University of Ulsan College of Medicine, Seoul; \\ 2Department of Pathology, Kyung Hee University Hospital at Gangdong, Kyung Hee University College of Medicine, Seoul; \\ ${ }^{3}$ Department of Otolaryngology, Asan Medical Center, University of Ulsan College of medicine, Seoul, Korea
}

\begin{abstract}
Hepatoid thymic carcinoma is an extremely rare subtype of primary thymus tumor resembling "pure" hepatoid adenocarcinomas with hepatocyte paraffin 1 (Hep-Par-1) expression. A 53-year-old man presented with voice change and a neck mass. Multiple masses involving the thyroid, cervical and mediastinal lymph nodes, and lung were detected on computed tomography. Papillary thyroid carcinoma was confirmed by biopsy, and the patient underwent neoadjuvant chemoradiation therapy. However, the anterior mediastinal mass was enlarged after the treatment whereas the multiple masses in the thyroid and neck decreased in size. Microscopically, polygonal tumor cells formed solid sheets or trabeculae resembling hepatocytes and infiltrated remnant thymus. The tumor cells showed immunopositivity for cytokeratin 7, cytokeratin 19, and Hep-Par-1 and negativity for $\alpha$-fetoprotein. Possibilities of germ cell tumor, squamous cell carcinoma, and metastasis of thyroid papillary carcinoma were excluded by immunohistochemistry. This report on the new subtype of thymic carcinoma is the third in English literature thus far.
\end{abstract}

Key Words: Hepatoid carcinoma; Thymus; $\alpha$-Fetoprotein; Hep-Par-1

Received: November 17, 2020 Accepted: March 10, 2021

Corresponding Author: Joon Seon Song, MD, PhD, Department of Pathology, Asan Medical Center, University of Ulsan College of Medicine, 88 Olympic-ro 43-gil, Songpa-gu, Seoul 05505, Korea

Tel: +82-2-3010-4548, Fax: +82-2-472-7898, E-mail: songjs@amc.seoul.kr

*These authors contributed equally to this work.

Hepatoid thymic carcinoma (HTC) is a rare subtype of thymic carcinoma and has been inserted in the 4th edition WHO classification of tumors of the lung, pleura, thymus and heart in 2015 [1]. It was first described by Franke et al. [2] in 2004 and only two cases have been reported until now [2,3]. This tumor was defined as a thymic carcinoma morphologically resembling hepatocellular carcinoma with immunoreactivity for hepatocyte paraffin 1 (Hep-Par-1) but immunonegativity for $\alpha$-fetoprotein (AFP), differentiating from hepatoid adenocarcinoma (HAC) of other organs [2]. Herein, we report an additional case of HTC.

\section{CASE REPORT}

A previously healthy 53-year-old man presented with voice change and a neck mass. On the neck computed tomography (CT) and chest CT, multiple masses involving bilateral lobes of the thyroid gland, cervical lymph nodes and left mediastinum were detected (Fig. 1). Papillary thyroid carcinoma with metastases to neck lymph nodes were confirmed by core needle biopsies, and the mediastinal mass showed poorly differentiated carcinoma. The patient underwent neoadjuvant chemoradiation therapy (radiation therapy, $70 \mathrm{~Gy} / 35$ fractions; cisplatin based chemotherapy for 3 cycles) under the impression of long lasting papillary thyroid carcinoma with anaplastic change. However, the anterior mediastinal mass was enlarged after the treatment whereas the thyroid and neck masses decreased in size. The patient underwent total thyroidectomy with radical neck dissection and mediastinal mass excision. Both thyroid masses were severely calcified, and the histologic features were those of classic papillary thyroid carcinoma. An ill-demarcated anterior mediastinal mass measured $6 \mathrm{~cm}$ and showed heterogeneous, tan colored, and firm cut surface (Fig. 2A). Microscopically, polygonal tumor cells formed solid sheets or trabeculae resembling hepatocytes and infiltrated remnant thymus. The tumor cells often 

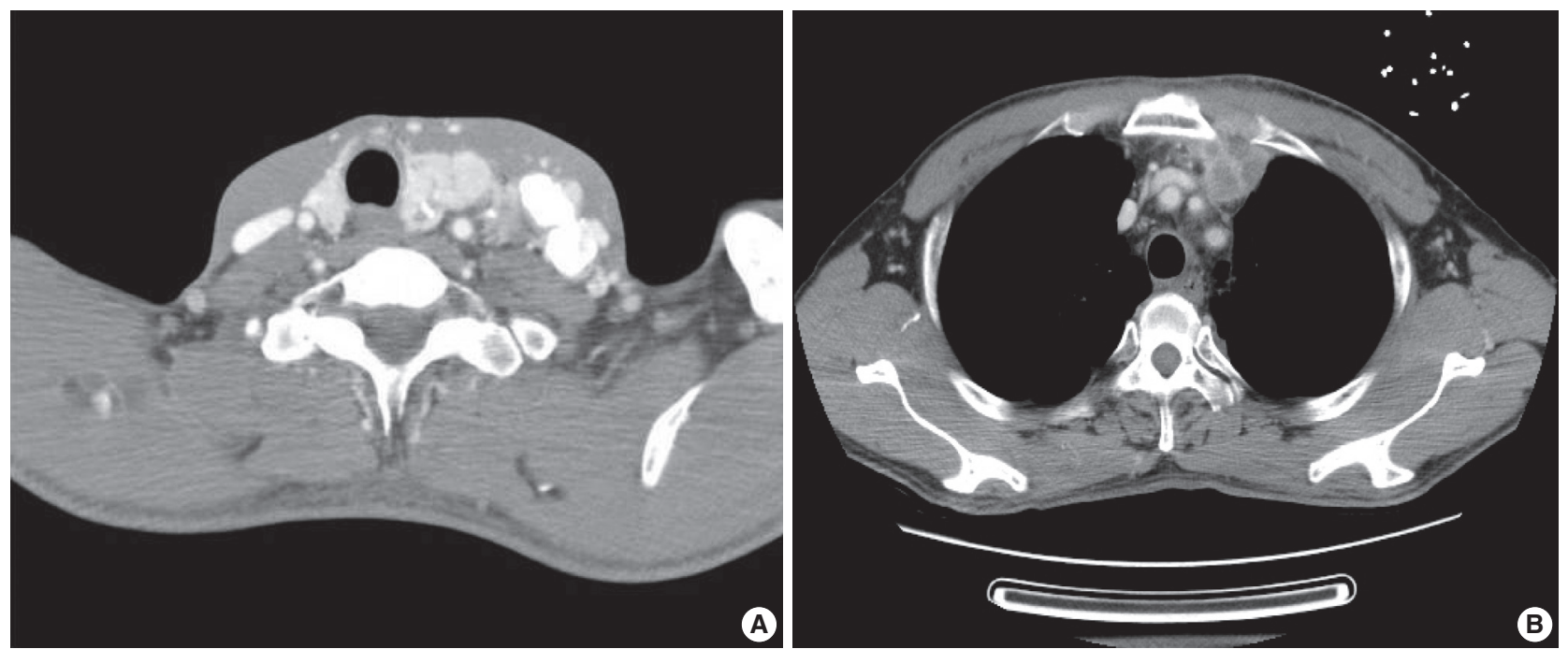

Fig. 1. Radiologic findings. (A) Neck computed tomography (CT) shows a large infiltrating mass with calcification in left thyroid lobe and multiple hypervascular masses in the left level II-VI, left supraclavicular area and right lower neck. (B) The chest CT shows anterior mediastinal mass, measuring $6 \mathrm{~cm}$.
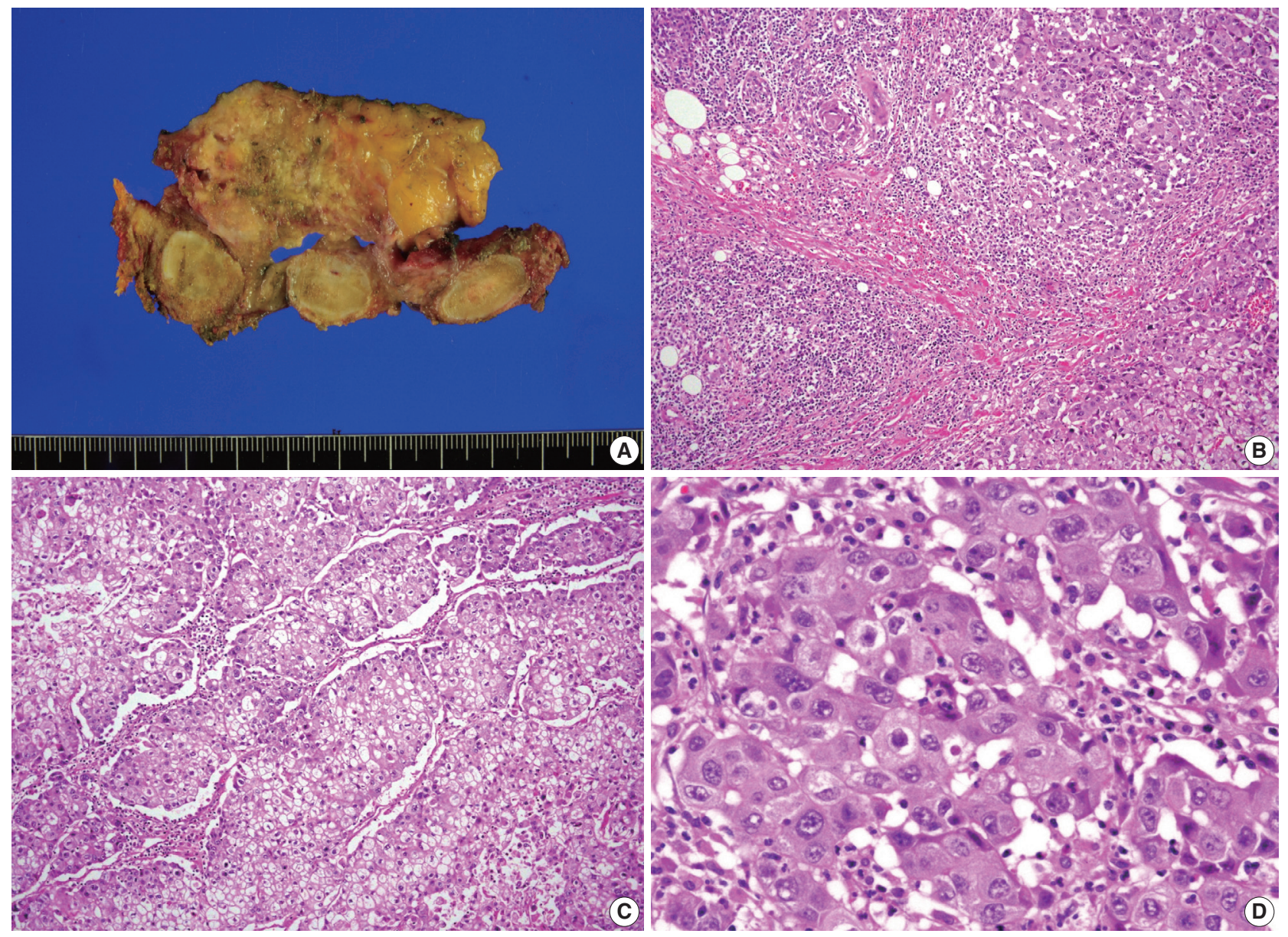

Fig. 2. Pathologic findings. (A) Grossly, an ill-demarcated anterior mediastinal mass, measuring $6 \mathrm{~cm}$ shows a heterogeneous, tan colored, firm cut surface. (B, C) Microscopically, the polygonal tumor cells formed solid sheet or trabeculae resembling hepatocytes and infiltrated remnant thymus. Part of the tumor shows clear cytoplasm. (D) The tumor cells show distinct cell borders, abundant eosinophilic cytoplasm, and pleomorphic vesicular nuclei. 

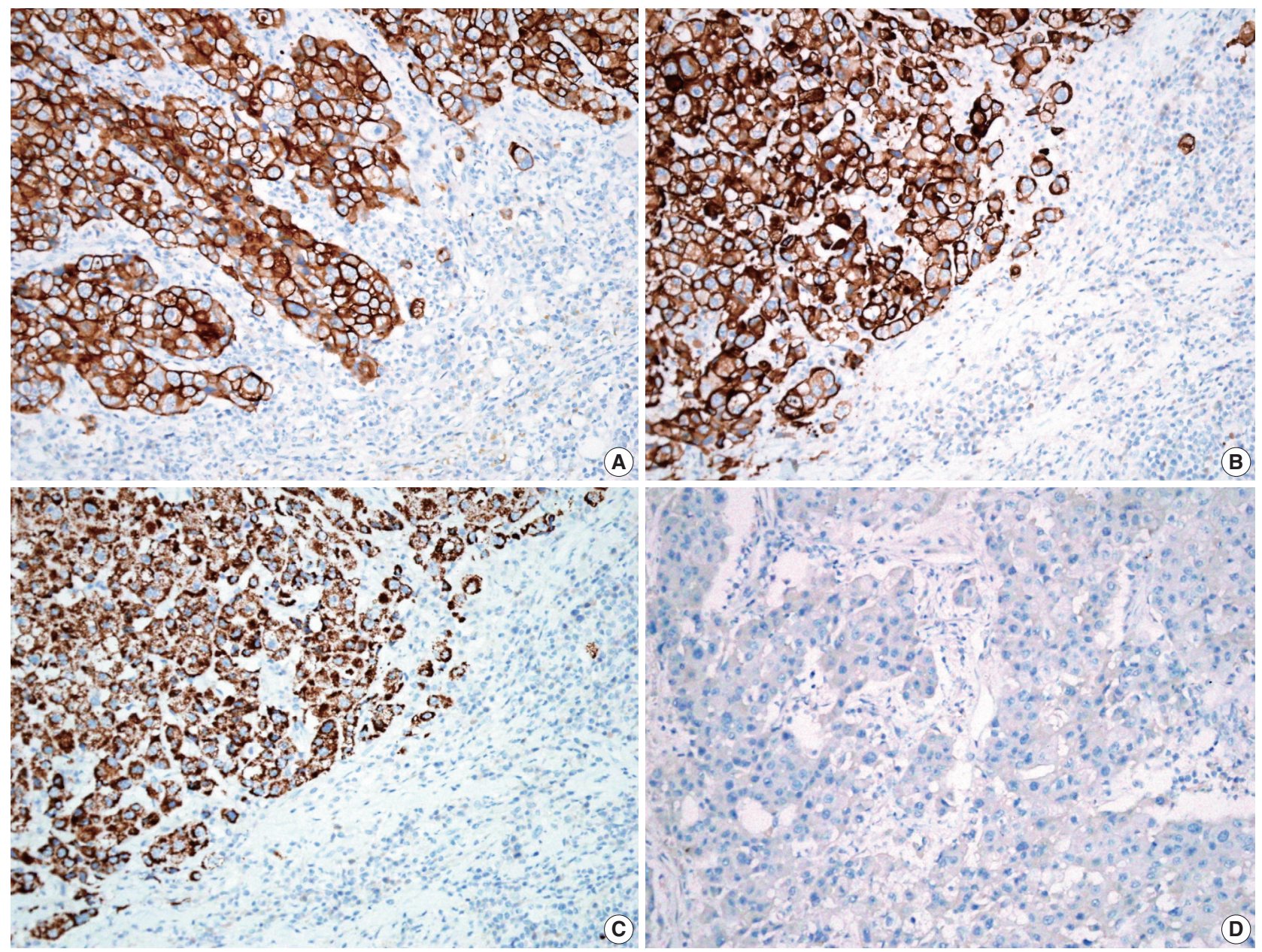

Fig. 3. Representative image of immunohistochemistry. The tumor cells show immune-positivity for cytokeratin 7 (A), cytokeratin 19 (B), and Hep-Par-1 (hepatocyte, C) and negativity for $\alpha$-fetoprotein (D) by immunohistochemistry.

showed clear cytoplasmic change (Fig. 2B-D), and immunopositivity for cytokeratin (CK) 7, CK 19 and Hep-Par-1 and negativity for AFP (Fig. 3). Possibilities of metastatic hepatocellular carcinoma, germ cell tumor, squamous cell carcinoma, anaplastic thyroid carcinoma, and NUT carcinoma were excluded by immunohistochemistry (IHC). The results of IHC are summarized in Table 1. The patient has been lost to follow-up after 6-month follow-up.

\section{DISCUSSION}

HTC is extremely rare and only two cases have been published in English literature thus far [2,3]. In brief, the patients were 70 -year-old female and 34-year-old male. The sizes measured $18 \mathrm{~cm}$ and $6.6 \mathrm{~cm}$. The patients underwent surgery with adjuvant chemoradiation therapy. The outcomes were not informative due to short follow-up periods or failure to follow-up.
The results of IHC staining showed similar profiles in all cases as follows: CK $7(+)$, CK $19(+/-)$, Hep-Par-1 (+), and AFP (-) (Table 2).

To exclude the possibility of anaplastic transformation of papillary thyroid carcinoma or poorly differentiated thyroid carcinoma, we performed IHC. Based on the results of IHC (paired box 8 [PAX-8] [-], thyroid transcription factor-1 [-], thyroglobulin $[-]$ ), we thought that this case was not of thyroid origin. It has been reported that immunoreactivity for PAX-8 in anaplastic thyroid carcinoma was $79 \%$ [4]. Histologic features, adjacent involved thymic tissue and immunoreactivity for HepPar-1 supported the diagnosis of HTC.

HAC has been reported in many other organs including the stomach, gallbladder, lung, uterus, and urinary bladder, etc. [5]. HTCs show distinct immunoprofiles that differentiate them from HAC. While $91.6 \%$ of HAC express AFP and $38.1 \%$ of HAC express Hep-Par-1, all HTCs are negative for AFP protein 
and positive for Hep-Par-1 although the cases are extremely rare. Hep-Par-1 is recognized as a mitochondrial antigen of hepatocyte and it is normally expressed in small intestinal epithelium and hepatocytes. Thus, it is highly sensitive (92\%) in diagnosing hepatocellular carcinoma [6]. However, our case had no hepatic mass, no evidence of hepatitis $\mathrm{B}$ and $\mathrm{C}$, and was immunoreactive for $\mathrm{CK} 7$ and CK 19, excluding the possibility of metastatic hepatocellular carcinoma.

$\mathrm{HAC}$ is a very aggressive neoplasm with metastasis at the time of diagnosis in a high proportion of patients and has a worse prognosis than more common types of tumors [7]. More than

Table 1. The results of immunohistochemical staining

\begin{tabular}{llll}
\hline \multicolumn{1}{c}{ Antibody } & Clone & Dilution & Results \\
\hline Cytokeratin 7 & Dako, Denmark & $1: 400$ & Positive \\
Cytokeratin 19 & Cell Marque, USA & $1: 100$ & Positive \\
Hepatocyte & Dako, Denmark & $1: 200$ & Positive \\
$\alpha$-Fetoprotein & Neomarkers, USA & $1: 200$ & Negative \\
TTF-1 & Novo, UK & $1: 200$ & Negative \\
Thyroglobulin & Dako, Denmark & $1: 2,000$ & Negative \\
Galectin-3 & Novo, UK & $1: 200$ & Negative \\
PAX-8 & Cell Marque, USA & $1: 50$ & Negative \\
SALL 4 & Biocare, USA & $1: 100$ & Negative \\
Oct 3/4 & Novo, UK & $1: 100$ & Negative \\
PLAP & Dako, Denmark & $1: 100$ & Negative \\
$\beta$-hCG & Cell Marque, USA & $1: 1,000$ & Negative \\
CD10 & Novo, UK & $1: 25$ & Negative \\
p63 & Novo, UK & $1: 20$ & Negative \\
HMB45 & DAKO, Denmark & $1: 50$ & Negative \\
TFE3 & Cell Marque, USA & $1: 50$ & Positive \\
Calretinin & Zymed, USA & $1: 400$ & Negative \\
WT1 & Dako, Denmark & $1: 100$ & Negative \\
Chymotrypsin & Chemicon (Millipore), USA & $1: 16,000$ & Negative \\
$\alpha 1$ antitrypsin & Santa Cruz, Germany & $1: 400$ & Negative \\
CD56 & NOVO, UK & $1: 25$ & Negative \\
CD30 & Dako, Denmark & $1: 25$ & Negative \\
CD5 & Novo, UK & $1: 200$ & Negative \\
NUT & Cell signaling, USA & $1: 100$ & Negative \\
c-erb2 & Dako, Denmark & $1: 500$ & Negative \\
\hline
\end{tabular}

TTF-1, thyroid transcription factor-1; PAX-8, paired box 8; SALL4, Sal-like protein 4 ; Oct $3 / 4$, octamer-binding transcription factor $3 / 4$; PLAP, placental alkaline phosphatase; $\beta$-hCG, beta-subunit of human chorionic gonadotropin; TFE3, transcription factor binding To IGHM enhancer 3; WT1, Wilms' tumour 1 . half of patients died within the first 12 months [5]. The 5-year survival rate of gastric HAC has been known to be significantly lower than that of conventional gastric cancer $(9 \%$ vs. $44 \%, \mathrm{p}=$ .001) [7]. The 5-year survival rate of thymic carcinoma ranges from $30 \%$ to $70 \%$, depending on the initial stage of disease [8]. HTC is also thought to have a poor prognosis, judging from the cases of HAC; however, it is hard to say definitively due to the small number of cases.

Histogenesis of this tumor is still unclear and the previous authors favored an endodermal origin rather than a germ cell origin [2,3], based on the hypothesis of endodermal origin of gastric and pulmonary hepatoid neoplasms $[9,10]$. Further study including genetic analysis is required to determine the histogenesis of this tumor.

In conclusion, we report this rare subtype of thymic carcinoma having peculiar characteristics of morphology and immunohistochemical features. In addition, being aware of this entity will contribute to collecting an adequate number of cases to lay the groundwork for identifying pathophysiology and establishing treatment in the near future.

\section{Ethics Statement}

This case was deemed exempt by the Asan Medical Center Institutional Review Board (IRB \#2020-1567). Informed consent was obtained from individual participant included in this study.

\section{Availability of Data and Material}

All data generated or analyzed during the study are included in this published article.

\section{Code Availability}

Not applicable.

\section{ORCID}

Ji-Seon Jeong https://orcid.org/0000-0002-4596-3658 Hyo Jeong Kang https://orcid.org/0000-0002-5285-8282 Uiree Jo https://orcid.org/0000-0001-6783-4016 Min Jeong Song https://orcid.org/0000-0002-5891-4329 Soon Yeol Nam https://orcid.org/0000-0002-8299-3573 Joon Seon Song https://orcid.org/0000-0002-7429-4254

\section{Author Contributions}

Conceptualization: JSS, JSJ, HJK. Data curation: JSJ, HJK, UJ, MJS, SYN.

Table 2. Summary of hepatoid thymic carcinoma reported in the English literature

\begin{tabular}{lccllll}
\hline Study & $\begin{array}{c}\text { Age }(\mathrm{yr}) / \\
\text { Sex }\end{array}$ & Size $(\mathrm{cm})$ & Location & \multicolumn{1}{c}{ Treatment } & Outcome & Immunohistochemistry \\
\hline Franke et al. [2] & 70/F & 18 & Thymus & Surgery+adjuvant RTx & AWD (22 mo) & CK7(+), CK19(+), HepPar-1(+), AFP(-) \\
Lee et al. [3] & 34/M & 6.6 & Thymus & Surgery+adjuvant CCRTx & FU loss (2 yr, AWD) & CK7(+), CK19(-), HepPar-1(+), AFP(-) \\
Present case & 53/M & 6.0 & Mediastinum & Neoadjuvant CCRTx+surgery & FU loss (6 mo, AWD) & CK7(+), CK19(+), HepPar-1(+), AFP(-) \\
\hline
\end{tabular}

F, female; RTx, radiation therapy; AWD, alive with disease; CK, cytokeratin; HepPar-1, hepatocyte paraffin 1; AFP, $\alpha$-fetoprotein; M, male; CCRTx, chemoradiation therapy; FU, follow-up. 
Methodology: JSS, JSJ. Project administration: JSS. Writing—original draft: JSS, JSJ. Writing—review \& editing: JSS, HJK. Approval of final manuscript: all authors.

\section{Conflicts of Interest}

JSS, a contributing editor of the Journal of Pathology and Translational Medicine, was not involved in the editorial evaluation or decision to publish this article. All remaining authors have declared no conflicts of interest.

\section{Funding Statement}

No funding to declare.

\section{References}

1. Travis WD, Brambilla E, Burke AP, Marx A, Nicholson AG. WHO classification of tumours of the lung, pleura, thymus and heart. 4th ed. Lyon: IARC Press, 2015.

2. Franke A, Strobel P, Fackeldey V, et al. Hepatoid thymic carcinoma: report of a case. Am J Surg Pathol 2004; 28: 250-6.

3. Lee JH, Kim H, Chae YS, Won NH, Choi JS, Kim CH. Hepatoid thymic carcinoma: a case report. Korean J Pathol 2009; 43: 562-5.

4. Nonaka D, Tang Y, Chiriboga L, Rivera M, Ghossein R. Diagnostic utility of thyroid transcription factors Pax8 and TTF-2 (FoxE1) in thyroid epithelial neoplasms. Mod Pathol 2008; 21: 192-200.

5. Su JS, Chen YT, Wang RC, Wu CY, Lee SW, Lee TY. Clinicopathological characteristics in the differential diagnosis of hepatoid adenocarcinoma: a literature review. World J Gastroenterol 2013; 19: 321-7.

6. Chu PG, Ishizawa S, Wu E, Weiss LM. Hepatocyte antigen as a marker of hepatocellular carcinoma: an immunohistochemical comparison to carcinoembryonic antigen, CD10, and alpha-fetoprotein. Am J Surg Pathol 2002; 26: 978-88.

7. Liu X, Cheng Y, Sheng W, et al. Analysis of clinicopathologic features and prognostic factors in hepatoid adenocarcinoma of the stomach. Am J Surg Pathol 2010; 34: 1465-71.

8. Engels EA. Epidemiology of thymoma and associated malignancies. J Thorac Oncol 2010; 5(10 Suppl 4): S260-5.

9. Ishikura H, Kirimoto K, Shamoto M, et al. Hepatoid adenocarcinomas of the stomach: an analysis of seven cases. Cancer 1986; 58: 119-26.

10. Ishikura H, Kanda M, Ito M, Nosaka K, Mizuno K. Hepatoid adenocarcinoma: a distinctive histological subtype of alpha-fetoproteinproducing lung carcinoma. Virchows Arch A Pathol Anat Histopathol 1990; 417: 73-80. 